\title{
Ueber Mikrophthalmus und Cataracta congenita vasculosa,
}

nebst einigen Bemerkungen über Drüsen der Chorioidea und Neubildung von Linsenkapsel.

Von

Dr. med. W. von Grolman, Assistent an der Universitäts-Augenklinik zu Giessen.

Hierzu Taf. XIII, Fig. 1-3.

Der im Nachfolgenden näher beschriebene Bulbus stammt aus der Sammlung der hiesigen Universitäts-Augenklinik. Für die gütige Ueberlassung desselben spreche ich Herrn Prof. von Hippel hiermit meinen besten Dank aus.

Das Präparat scheint mir nicht nur in seiner Eigenschaft als Mikrophthalmus, sondern auch wegen höchst auffallender Veränderungen an der Linse ein allgemeineres Interesse zu verdienen. Ich gebe zunächst die aus der Krankengeschichte entnommenen Data:

Die 12 jährige Marg. B. wurde im Mai 1879 von den Eltern in die hiesige Poliklinik geführt. Sie hatte ein kleineres rechtes Auge mit zur Welt gebracht, das von vornherein eine weissliche Trübung im Pupillargebiete zeigte, die noch damals bestand und zuerst am dritten Tag nach der Geburt des Kindes beobachtet wurde. Bei anstrengender Arbeit soll dasselbe zuweilen gethränt haben. Seit einigen Monaten bereits klagte die Kleine über Schmerzen und andere sympathische Erscheinungen am linken Auge. Ein grauer Schimmer schwebte beständig vor demselben. 
Die Untersuchung ergab ein in allen Durchmessern yerkleinertes rechtes Auge mit klarer normal gewölbter Cornea, deren Grösse der des ganzen Bulbus entspricht. Vordere Kammer ziemlich flach. Iris in der Mitte etwas vorgetrieben, ihr Pupillarrand überall mit der kataraktösen Linse verwachsen. Lichtschein fehlt; Druckempfindlichkeit des Corpus ciliare. Motilität nicht im Geringsten gestört. - L. A. Myopie $1 / 14 \mathrm{~S}=1$. - Der Bulbus wurde enucleirt und in Müller'scher Flössigkeit conservirt. Makroskopisch repräsentirt sich derselbe als vollkommen wohlgebildet; abgesehen yon der Verkleinerung ist äusserlich keine pathologische Veränderung wahrzunehmen. Sagittaldurchmesser: $14,5 \mathrm{~mm}$, Aequatorialdurchmesser: $15,5 \mathrm{~mm}$, Basis der Cornea 7,5 mm. Nach Theilung des Präparates im horizontalen Meridian fällt die anscheinend wohlgebildete Linse, an der sich zunächst keine Dislocation erkennen lässt, durch ihre schneeweisse Farbe auf. Von ihrem hinteren Pol setzt sich bis zur Mitte des Opticuseintritts ein weisser Strang nach hinten fort. Die Netzhaut scheint total abgelöst und trichterförmig gefaltet.

Nach Einbettung des Präparates in Celloidin zerlegte ich den grösseren Theil der unteren Hälfte in eine Reihe von Serienschnitten, die mit Hämatoxylin und Eosin gefärbt und in Canadabalsam eingeschlossen wurden.

Die mikroskopische Untersuchung ergab folgenden Befund: Cornea und Sclera zeigen, abgesehen von einer der Grösse des Bulbus entsprechenden Dickenabnahme keine weitere Anomalie; das Gleiche gilt von dem Sehnerven und seiner Scheide, an dem nur die stark entwickelte Centralarterie auffällt.

Die Retina ist überall von der Pigmentschicht abgelöst und bildet die bekannte Figur der Windenblüthe. Eine grössere Continuitätstrennung konnte ich nicht in derselben nachweisen. Als wohlbegrenztes Band hebt sich die innere Körnerschicht von dem übrigen Gewebe ab. Innerhalb derselben sieht man überaus zahlreiche rundliche Lücken, die bei schwacher Vergrösserung ganz fein granulirt aussehen, während unter Anwendung stärkerer Objective erkannt wird, dass sie meistens einen Gefässquerschnitt enthalten. Die Wandungen dieser Gefässe sind grösstentheils stark verdickt und glasig degenerirt. In nicht wenigen der beschriebenen Lücken ist ein Gefässquerschnitt nur noch mit Mühe nachzuweisen, während in wieder anderen jede Spur eines solchen fehlt. Es liegt die Vermuthung nahe, dass auch diese Stellen einst gefässhaltig waren. 
Ueber Mikrophthalmus und Cataracta congen. vasculosa. 189

Als gelblich tingirter Streifen schliesst sich an die inneren Körner die Zwischenkörnerschicht an; obschon meist wohlbegrenzt, ist sie doch an manchen Scbnitten wiederum kaum nachzuweisen. Die äusseren Körner bilden nur eine wirre Masse, die ebenfalls zahlreiche Gefässe aufweisst. Von Stäbchen und Zapfen sind nur vereinzelte Rudimente aufzufinden. Die inneren Theile der Netzhaut zeigen durchweg bindegewebige Degeneration. In allen Schichten findet man unregelmässig vertheilt einzelne Pigmenthaufen. An einer Stelle der Retina ist eine dünne Lamelle in Form einer enorm grossen Blase abgehoben, die fast bis zur Pigmentschicht beranreicht.

In dem subretinalen Raum finden sich nur einzelne körnige Gerinnungen, und deckt sich auch der Hauptinhalt des Retinaltrichters im Wesentlichen mit diesem Befund. Ausserdem erkennt man jedoch, dass der bereits oben erwähnte weisse Strang als die persistirende Arteria hyaloidea aufzufassen ist. Sie zeigt eine sehr starke kernreiche Adventitia und ein auffallend kleines Lumen. Ehe ich auf ihr merkwürdiges Verhalten in Bezug auf die Linse eingehe, möchte ich noch den Befund am Uvealblatt erwähnen. Das Stroma der Chorioidea, die der Sclera überall anliegt, zeigt nur geringgradige entzündliche Infiltration, der Ciliarkörper erscheint sogar ganz normaI. Die Iris ist gleichfalls frei von jeder Entzündung; das Stroma derselben schlägt sich um den Pupillarrand des Pigmentblattes herum und bildet auf der Oberfläche der Linse eine zweite dünnere Lage, die sich nach innen zu durch einzelne Pigmentzellen noch in der Näle des Aequators verräth, während sie nach aussen mit einem stumpfen Rand abschliesst.

Die überall der Chorioidea anliegende Pigmentepithelschicht ist mit zahllosen sogenannten Chorioidealdrusen übersäet. Da Gama Pinto ${ }^{1}$ ) hat sie gleichfalls an einem Kaninchenmikrophthalmus beobachtet und ausführlicher beschrieben. Er spricht sich entschieden für ihre Entstehung durch Degeneration der Pigmentzellen aus, während Leber ${ }^{2}$ ), gegen den sich Pinto an der erwähnten Stelle wendet, sie für eine Cuticulabildung der Pigmentzellen ansieht.

Eine einheitliche Erklärung für die Genese dieser Gebilde scheint mir überaus schwierig zu sein. Morphologisch möchte ich zwei verschiedenen Arten derselben unterscheiden. Gemeinsam war sämmtlichen Drusen meiner Präparate ein theils ein-
1) No. 1, p. 90 .
) No. 2, p. $308 \mathrm{ff}$. 
facher, theils doppelter oder mehrfacher continuirlicher Epithelüberzug und die scharfe Abtrennung von der Basalmembran der Chorioidea, die stets unversehrt unter ihnen herzog. In einzelnen etwas dickeren Schnitten fand ich die Drusen in toto vor, so dass ich eine Flächenansicht der mir zugekehrten Halbkugel gewinnen konnte; dabei fiel mir zuweilen auf, dass der ganze Epithelüberzug von normaler Beschaffenheit war.

Wesentlichere Unterschiede zeigte dagegen der innere Bau der Excrescenzen. Weitaus die meisten enthielten eine fein gekörnte, zuweilen fast homogene Masse. Bei starker Vergrösserung gelingt es leicht, diese in einzelne Schollen aufzulösen, innerhalb welcher ich an absichtlich etwas mit Hämatoxylin äberfärbten Präparaten Kernreste in allen Stadien des Zerfalls nachweisen konnte. In Fig. 2 habe ich rechts eine Druse wiedergegeben, deren Zusammensetzung aus degenerirten Zollen mit theilweise noch wohlerhaltenen Kernen mit Leichtigkeit zu erkennen ist, wäbrend in der zur Linken nur ganz vereinzelte Reste von Zellgrenzen und ein einziger Kern zu. bemerken ist.

Eine kleine Minderzahl zeigte den bekannten zwiebelartig geschichteten Bau und eine völlig durchsichtige glasige $\mathrm{Be}-$ schaffenheit.

Letzteren Fällen gegenäber liegt der Gedanke an eine Entstehung durch Cuticularbildung wohl sehr nahe; dagegen dürfte es unmöglich sein für die in Fig. 2 wiedergegebenen Befunde diese Anuahme festzuhalten. Andererseits scheint mir die zwiebelartige Schichtung nur schwer durch degenerativ zerfallende Zellen erzengt werden zu können. Man muss denn gerade annehmen, dass durch den Druck der im Inneren immer stärker quellenden Massen eine verschiedene Dichtigkeit der inneren und äusseren Schichten zu Stande kommt, deren ungleiche Brechkraft dem durchfallenden Lichte gegenüber in den concentrischen Ringen sich optisch geltend macht.

Eine Fülle der interessantesten Veränderungen zeigt die Linse und ihre Kapsel. Ich verweise zum leichteren Verständniss der Beschreibung auf die in grösserem Maassstabe beigegebene Abbildung. Die änssere Form schliesst sich zwar ungefähr der der normalen Linse an, zeigt aber im Einzelnen doch beträchtliche Abweichungen. Weniger kommt dabei die Vergrösserung des sagittalen Durchmessers, als die unregelmässige Krümmung der Oberfläche in Betracht. Die vordere Kapsel zunächst ist stark und höchst ungleichmässig verdickt. Sie 
reicht nicht ganz bis zum Aequator, wo sie sich zipfelartig einschlägt, um keine Fortsetzung auf der Hinterfläche der Linse zu finden. An vielen Stellen insbesondere an den Enden finden sich kolbige Hohlräume, die mit meist wohlerhaltenen oder nur wenig gequollenen Epithelzellen ausgefüllt sind. An manchen Schnitten lässt sich jedoch nachweisen, dass die ganze Kapsel aus zwei Blättern besteht, zwischen denen sich überall vereinzelte Epithelzellen finden, die nur an den erwähnten Stellen zu grösseren Agglomeraten angehäuft sind. Ich komme später nochmals auf diesen Befund zurück. Die Hinterfläche der Linse ist mit einer fein fibrillären bindegewebigen Masse überzogen, die in keinem erkennbaren Zusammenhang mit der vorderen Kapsel steht; sie zeigt sehr ungleiche Dickenverhältnisse und sendet zipfelartige Fortsätze theils zwischen die benachbarten abgelosten Retinafalten, theils nach der Hinterfläche des Corpus ciliare. Nach hinten setzt sie sich unmittelbar in die verdickte Scheide der Hyaloidea fort. Bei ihrem Uebergang in diese treten sofort zablreiche Kerne in ihr auf. An ihrer Innenfläche lässt sich nur hie und da auf kürzere Strecken eine gleichmässig dicke Lamelle abgrenzen, welche möglicherweise als Rest der eigentlichen Hinterkapsel $\mathrm{zu}$ betrachten ist.

Sehen wir uns nun das Innere des so umschriebenen Hohlraumes etwas näher an, so finden wir als einzige noch in ihrer Abstammung erkennbaren Reste der Linsenfasern jene tropfenartigen Gebilde, wie sie in jeder Katarakt zu beobachten sind. Sie sehen da, wo sie in grösseren Gruppen auftreten Fettgewebe auffallend ähnlich. Im Allgemeinen unregelmässig vertheilt, sind sie doch in den hinteren Parthieen der Linse häufiger als in der Nähe der vorderen Kapsel zu treffen: Bei der in der Figur wiedergegebenen Vergrösserung sind sie ganz durchsichtig und homogen, während man bei Anwendung stärkerer Objective eine feine Granulirung ihrer peripheren und eine unregelmässige Zeichnung ihrer centralen Theile erkennt (Fig. 3).

Eingeschlossen sind sie von einem feinfaserigen Gewebe, dessen einzelne krausgewundene Fibrillen in den peripheren Theilen ein dichteres Netzwerk bilden als im Centrum. Im Allgemeinen folgt die Richtung derselben der normaler Linsenfasern; nur in der Nähe der vorderen Kapsel sind sie mehr parallel zu dieser angeordnet. Hie und da findet man weisse Blutkörperchen in dem Gewebe zerstreat, die sich an einzelnen Stellen sogar zu dichteren Haufen zusammenfinden. 
Als drittes Element kommen in allen Präparaten zahlreiche Gefässe vor, von denen oft reich verzweigte Theile immerhalb eines Schnittes beobachtet werden. Das grösste derselben geht in genau sagittaler Richtung vom hinteren Pol der Linse aus. Sein Inneres birgt eine grössere Menge rother Blutkörperchen. Meist jedoch zeigen diese Gefässe starke Wandungen und ein nnverhältnissmässig kleines Lumen, so dass sie oft nur eine perlschnurartig angeordnete Reihe Blutkörperchen enthalten (Fig. $1 \mathrm{f}$ ). Wo sie im Querschnitt getroffen sind, kann man beobachten, dass die umgebenden Fibrillen die Scheide zur Hälfte concentrisch umfassen, um nach der vorderen Seite hin büschelförmig auszustrahlen ( $\mathrm{g}$ in der Fig.).

Ueber die Genese des Mikrophthalmus hat Hess ${ }^{1}$ ) im vorigen Jahre eine Arbeit veröffentlicht, in der er auf Grund seiner Untersuchungen von fünf mikrophthalmischen Bulbis sehr energisch wieder für die ältere Anschaung eintritt, welche diese Anomalie als eine Hemmungsbildung aufgefasst wissen will; er steht nur insofern im Gegensatz zu $\mathrm{Manz}^{2}$ ), als er den primären Grund nicht in einer übermässigen Gefässentwickelung des durch den Augenblasenspalt hereinwachsenden Mesoderms, sondern in einer Bindegewebshypertrophie dieser Theile sieht.

Bei diesem wie den gleich näher zu besprechenden Erklärungsversuchen wird stets ein Zusammenhang des Mikrophthalmus mit Colobom, sei es der Iris, sei es der Chorioidea oder beider vorausgesetzt.

Ganz anders als obige Forscher suchte bekanntlich Deutschmann ${ }^{3}$ ) die Entstehung eines von ihm beobachteten Vikrophthalmus beim Kaninchen zu erklären. Er fand in der Gegend des Coloboms ausgesprochen entzündliche Veränderungen des Gewebes und schliesst daraus, dass ,der grösste Theil, wenn nicht alle diese Bulbuscolobome" als Folgen einer solchen in sehr früher Zeit entstandenen intrauterinen Entzündung anzusehen seien. Ihm hatten sich in rascher Folge Höltzke $\mathrm{e}^{4}$ ), Falchi ${ }^{5}$ ) u. A. angeschlossen.
1) No. 3, p. $147 \mathrm{ff}$.
2) No. 4, p. 68 .
3) No. 5, p. 112.
5) No. 7, p. 213.

4) No. 6, p. 153 . 
Ueber Mikrophthalmus und Cataracta congen. vasculosa. 193

So wenig ich leugnen kann, dass es mir sehr gewagt erscheint, wenn Hess an dem Bulbus seines dritten Falles, der neben totaler Netzhautablösung auch ausgedehnte Hornhautinfiltration und Vascularisation zeigte, alle entzündlichen Erscheinungen in Abrede stellt, so sehr stimme ich ihm bei, insofern er der Meinung derer entgegentritt, die mit Deutschmann statt des ,vagen Begriffs der Hemmungsbildung“ das soeben beschriebene Causalitätsverhältniss annehmen möchten. Schon allein die häufige Doppelseitigkeit, sowie das gleichzeitige Auftreten anderer Bildungsfehler des ganzen Organismus legten doch die Unwahrscheinlichkeit primärer Entzündungen als Ursache des Coloboms und damit des Mikrophthalmus ziemlich nahe. In gleichem Sinne spricht der Umstand, dass bei Cochinchinahühnern regelmässig ein Colobom des Ciliarkörpers beobachtet wird. ${ }^{3}$ )

Sind wir aber durch die Deutschmann'sche Entzündungstheorie dem Verständniss dieser merkwürdigen Missbildungen auch nur um ein noch so geringes Maass näher gerückt, wie jener Autor zu glauben scheint? Warum entsteht gerade an jener verhängnissvollen Stelle im fötalen Bulbus mit Vorliebe eine ganz lokalisirte Entzündung?

Mir scheint diese Thatsache, die sich nicht leugnen lässt, nur dann verständlich zu sein, wenn man annimmt, dass etwa durch eine abnorme Nährmaterialvertheilung die rechtzeitige Ruickbildung der in der Spalte eingelagerten Gefässe und mesodermalen Gewebsschichten unterbleibt, wodurch die secundäre Augenblase bei ihrer Tendenz sich zu schliessen einen Widerstand in dem zwischengelagerten $\mathrm{Me}-$ sodermtheil findet, der naturgemäss sehr leicht zu einer entzündlichen Reizung und deren Folgen führen wird.

So wenigstens dürften sich die Vorgänge abgespielt haben in den Fällen, wo die Entzündung auf die Gegend

1) No. 8, p. 24 (auch von Hess für seine Ansichten jedoch in anderem Sinne verwerthet).

จ. Graefe's Archiv für Ophthalmologie. XXXV. 3. 
des Spaltes beschränkt erscheint. Auders steht die Sache dann, wenn eine allgemeine Entzündung der verschiedenen Bulbustheile vorliegt oder überhaupt kein Zusammenhang mit unvollkommener Rückbildung des gefässhaltigen Mesodermfortsatzes nachzuweisen ist. Ausgedehntere entzündliche Vorgänge haben sich in unserem Bulbus zweifellos abgespielt. Es fragt sich nur, ob dieselben bereits während der embryonalen Periode oder erst nach der Geburt ihren Anfang genommen. In dem erwähnten Falle Falchi's lagen ähnliche Verhältnisse vor. Das Auge war wegen Schmerzhaftigkeit im 29 ten Lebensjahre der Trägerin enucleirt worden. Während der Autor seinen Befund zu Gunsten der Entzündungstheorie anwenden will, weist Hess') diese Ansicht zurück, indem er annimmt, dass jene Veränderungen post partum entstanden seien.

Was nun meinen Fall betrifft, so halte ich es für ziemlich schwierig, in dieser Beziehung eine sichere Entscheidung zu treffen. Auf den ersten Blick scheint zwar die Aunahme, dass der Mikrophthalmus entzündlichen Vorgängen seinen Ursprung verdanke, ungemein viel für sich zu haben. Während doch, so wird man sich sagen, in den iibrigen Fällen die gleichzeitige Anwesenheit eines Coloboms trotz vorhandener Entzündung die Bedeutung dieser in genetischer Beziehung zweifelhaft macht, fällt dieses Dilemma hier einfach weg. In der That unterscheidet sich der von mir beschriebene Bulbus von fast allen anatomisch untersuchten Mikrophthalmen durch die Abwesenheit jener Anomalie. $\mathrm{Manz}^{2}$ ) äussert sich über diesen Punkt am Schlusse seiner Besprechung der Genese des Mikrophthalmus folgendermassen: ..... Es soll damit nicht gesagt sein, dass der Mikrophthalmus keine andere Ursache haben könne als das Colobom; es können gewiss auch andere Ereignisse daran Schuld sein, die uns bei dem geringen vorliegenden anatomischen Material noch unbekannt sind";
I) No. 1.
3) No. 4, p. 134. 
Ueber Mikrophthalmus und Cataracta congen. vasculosa. 195

und Arlt ${ }^{1}$ ) sprach sich noch im Jahre 1885 dahin aus, dass jeder Mikrophthalmus mit Colobom verbunden sei. Sehe ich von den Fällen $a b$, in denen einzelne Theile des Bulbus ganz fehlten, wie z. B. dem dritten von Hess beschriebenen, so wüsste ich nur den von dem gleichen Autor angeführten "reinen Mikrophthalmus", der jeder weiteren Missbildung entbehrte und den ron $\mathrm{Falchi}^{2}$ ), sowie den meinigen zur Widerlegung der Arlt'schen Ansicht anzuführen.

Statt des Coloboms finden wir in unserem Bulbus eine persistirende Art. hyaloidea. Eine solche wird neben gleichzeitigem Colobom von zahlreichen Autoren erwähnt. Ich verweise auf die Fälle ron Hänel ${ }^{3}$, $\mathrm{Hess}^{4}$ ) u. A. Becker ${ }^{5}$ ) sagt sogar: "Das Persistiren der Art. hyaloidea und mindestens eines Theils der gefässhaltigen Linsenkapsel ist die Regel." Nun kann man freilich nicht behaupten, dass eine restirende Glaskörperarterie an sich etwas mit dem verzögerten Schluss der Augenspalte zu thun habe. Allein der Umstand, dass sie fast immer bei gleichzeitigem Vorhandensein von Mikrophthalmus und Colobom beobachtet wird, legt doch den Gedanken nahe, dass da, wo das Colobom zwar fehlt, aber der Mikrophthalmus vorhanden ist, ähnliche Processe zu ihrer Persistenz gefuihrt haben, als in jenen Fällen von Mikrophthalmus, wo sich die Augenspalte nicht völlig geschlossen hat. Zur Stütze dieser Ansicht sei mir ein Vergleich mit dem ersten Falle von Hess gestattet: Mikrophthalmus ohne Spur einer Entziindung, isolirtes kleines Iriscolobom, Arteria hyaloidea. Niemand zweifelt, dass die Ursache aller dieser Missbildungen der verzögerte Schluss der secundären Augenblase war; und doch, wäre die Schliessung derselben nur noch wenige Millimeter vorwärts gerückt, so hätten wir nahezu den gleichen Befund wie im vorliegenden Fall: Mikrophthalmus, Art. hyaloidea, kein Colobom.

1) No. 9 citirt nach dem Referat von Manz in Michels Jahresbericht 1885, p. 225.

2) No. 7 .

3) No. 10 .

4) No. 1, Fall 1 und 3 .

5) No. 11, p. 126. 
Deshalb glaube ich, darf man auch für diesen einen Zusammenhang mit mangelbafter Rückbildung der mesodermalen gefässtragenden Theile nicht ganz zurückweisen. Durch den Kampf der gegen das Mesoderm andrängenden sccundären Augenblase könnten die weiteren Störungen, welche ein normal grosses Wachsthum verhinderten, und zu ausgedehnten entzündlichen Erscheinungen führten, entstanden sein.

Aus den angeführten Gründen bin ich der Ansicht, dass für unseren Bulbus die Frage, ob ein Entzündungsprodukt oder eine Hemmungsbildung vorliegt, nicht mit Sicherheit zu entscheiden ist.

Wenden wir nun unsere Aufmerksamkeit nochmals den VerhäItnissen an der Linse zu. Was zunächst das Gefässsystem derselben anbelangt, so scheint es mir nicht zweifelhaft, dass es als eine directe Fortsetzung der Art. hyaloidea anzusehen ist. Der Bulbus war leider durch den langen Aufenthalt in Alkohol beträchtlich geschrumpft und dabei genau in der Mitte getheilt. So kam es, dass die ursprünglich in dem gleichen positiven Meridian gelegenen Theile sich in einer Bogenlinie aneinander reihten und deshalb keine eigentlichen Horizontalschnitte, sondern lauter Schrägschnitte erzielt wurden. Dies der Grund, warum ich in keinem Präparat eine directe Verbindung des innerhalb des Kapselsacks gelegenen Gefässnetzes mit der Art. hyaloidea nachweisen konnte. Dennoch muss man mit absoluter Sicherheit folgern, dass der in der Fig. 1 mit $d$ bezeichnete Gefässstamm seinen Ursprung aus der Hyaloidea nimmt, und ebenso geht aus der Art der Verzweigung der übrigen intrakapsulären Gefässe ihr Zusammenhang mit $d$ hervor. Gefässe innerhalb des verletzten Kapselsacks sind mehifach beobachtet worden, jedoch wie es scheint nur in Verbindung mit gleichzeitiger Knochenbildung. Wenigstens begegnete ich keinem andersartigen sicher gestellten Fall in der Literatur. Auch Wedl und $B_{0} \mathbf{c k}^{1}$ ) erwähnen nichts

1) No. 12, S. 170 . 
Ceber Mikrophthalmus und Cataracta congen. vasculosa. 197

hierher gehöriges. Jedenfalls darf ich so viel behaupten, dass alle bisher mitgetheilten Befunde von Vascularisation des intracapsulären Gewebes genetisch durchaus verschieden von dem meinigen waren. Stets stammten die Gefässe aus dem benachbarten Corpus ciliare, während hier eine directe abnorme Fortsetzung der embryonalen Glaskörpergefässe in das Innere der Linse stattgefunden hat. Gemeinschaftlich dürfte nur Allen die entzündliche Ursache und die vorausgegangene ausgedehnte Verletzung des Kapselsackes sein. Das an die Stelle der hinteren Kapsel getretene Gewebe spreche ich für ein Umwandlungsprodukt des Glaskörpers an. Aehnlicher Herkunft dürfte auch das feinfaserige Netzwerk des Kapselinneren sein. Während es freilich nicht selten, wie dies auch die beigegebene Figur andeutet, durch einen kleinen Zwischenraum von der Pseudokapsel abgesetzt erscheint, so lässt sich doch wieder an anderen Stellen deutlich erkennen, wie es direct aus dieser entspringt (siehe Fig. 3).

Noch ein paar Worte möchte ich über die Veränderungen an der vorderen Kapsel hinzufügen. Wucherungen von Kapselzellen, die aussen und innen von Kapsel begrenzt waren, sind öfters beobachtet worden. Becker ${ }^{1}$ ) giebt in seinem Atlas mehrere hierauf bezügliche Abbildungen. Durch genaue Messungen will er festgestellt haben, dass in seinen Fällen die Summe der Dicken der. äusseren (sehr starken) und inneren (sehr dünnen) Lamelle stets genau gleich dem Durchmesser der unversehrten Kapsel in der Nachbarschaft gewesen sei. Er folgert nun, dass es sich nicht um eine Neubildung von Kapsel handeln könne, sondern nur um eine Wucherung der Zellen zwischen den einzelnen Schichten der Kapsel, in die sie nach Erosion der innersten Lamellen eingedrungen seien, um sich dort zu verbreiten. Diese Auffassung steht im Einklang mit der Ansicht derer, die die Kapsel als ein Produkt des Mesoderms und nicht als eine Cuticularbildung angesehen wissen wollen.

') No. 11, Abbild. S. 59/60, Text S. 76. 
Anders liegen die Verhältnisse in meinem Fall. Schon sin Blick auf die Abbildung genügt, um die Idee einer Spaltung der Kapsel als höchst unwahrscheinlich zurückweisen zu können. Die stets wechselnde Dicke, die ungefähre Gleichheit der inneren und äusseren Lamelle, die knopfförmigen Zellenanhäufungen am äquatorialen Ende der Kapsel lassen diesen Gedanken nicht aufkommen, Völlig undenkbar aber ist es, dass die ursprüngliche normale an der Innenseite der Kapsel gelegene Epithelschicht gänzlich verloren ging, während eine von ihr ausgegangene Wucherung zwischen die Lamellen der ersteren sich gleichmässig in der ganzen Ausdehnung derselben, meist dazu noch in nur ein- oder zweifacher Lage verbreitet habe. Wir müssen deshalb die vorliegende Veränderung als einen exquisiten Fall von Neubildung der Kapsel durch deren Zellen ansehen. In der letzten Abtheilung des Archivs für Ophthalmologie hat Wagenmann ${ }^{1}$ ) ganz analoge Beobachtungen über „Neubildung glashäutiger Substanz" veröffentlicht. Auch or hat eine doppelte Kapsel beobachtet und nimmt nicht nur für sie die gleiche Entstehung an, sondern will auch Becker's Beobachtung so gedeutet wissen, dass an den scheinbar einfachen Stellen sich nur die beiden Lamellen ungemein dicht aneinander gelegt hätten. Obgleich mir selbst an den ersten Schnitten, die ich untersuchte, die Kapselverdoppelung da entging, wo nur vereinzelte Zellen zwischen den beiden Blättern vorhanden waren, so sehe ich doch nicht ein, warum auch nicht der von Becker angenommene Modus einer Kapselverdoppelung vorkommen kann, zumal es doch gewiss unwahrscheinlich ist, dass einom so gewiegten Beobachter zwei nur angelegte Lamellen stets als einfache Kapsel imponirt haben sollten.

1) No. 13 .

Giessen, 20. Juni 1889. 


\section{Literaturverzeichniss.}

1. Da Gama Pinto, Mikrophthalmus congenitus beim Kaninchen. Archiv für Augenheilkunde XIII.

2. Leber, Ueber Amyloiddegen. der Conjunct. Archiv für Ophthalmologie XXV, 1.

3. Hess, Zur Pathogenese des Milkrophthalmus. Archiv für Ophthalmologie XXXIV, 3,

4. Manz, Missbildungen des Auges. Graefe-Sämisch Handb. Bd. II.

5. Deutschmann, Zur pathologischen Anatomie des Iris- und Aderhautcoloboms, als Grundlage eines Erklärungsversuchs der sogen. Hemmungsbildung überhaupt. Klinische Monatsblätter XIX, 101.

6. Höltzke, Mikrophthalmus und Colobom von einem Kaninchen. Archiv für Augenheilkunde XII.

7. Falchi, Microftalmo congenito. Annali di Ottalmologia XIII.

8. Lieberkühn, Beiträge zur Anatomie des embryon. Auges. Archiv für Anatomie und Physiologie 1879.

9. Arlt, Ueber Mikrophthalmus und Anophthalmus. Anzeiger der k. k. Gesellschaft der Aerzte in Wien, citirt nach dem Referat von Manz in Michels Jahrbuch 1885.

10. Hänel, Ein Fall von Arteria hyal. persistens. Persistenz des Kölliker'schen Mesodermfortsatzes und Iriscolobom. Inaugural-Dissertation München 1886.

11. Becker, Zur Anatomie der gesunden und kranken Linse. Wiesbaden 1883.

12. Wedl und Bock, Textbuch zum Atlas der pathologisehen Anatomie des Auges.

13. Wagenmann, Ueber Neubildung glashäutiger Substanz im Ange. Archiv für Ophthalmologie XXXV, 1. 


\title{
Figuren-Erklärung.
}

\section{Tafel XIII.}

\author{
Figur 1 .
}

Linse aus einem mikrophthalmischen Bulbus.

a Aeusseres

$a_{1}$ Inneres

Blatt der vorderen Kapsel.

$b$ Pigmentirte Iriszellen auf der Vorderfläche der Linsenkapsel.

$c$ Reste der Linsenfasern.

d Gefässstamm am hinteren Pol der Linse, mit rothen Blutkörperchen angefüllt (Fortsetzung der Art. hyal.).

$f$ Einfache Reihe rother Blutkörperchen im Innern der Gefässe.

$g$ Gefässquerschnitt, um den sich die benachbarten Fibrillen herumschlingen.

$h$ Ansammlung weisser Blutkörperchen.

$i$ Arteria hyaloidea.

$k$ Abgelöste Netzhaut.

\section{Figur 2.}

Zwei Drusen des Pigmentepithels.

a Epithelüberzug.

$b$ Degenerirte Epithelzellen mit absterbenden Kernen.

c Basalmembran der Chorioidea.

Figur 3.

Ein Stück der Pseudokapsel von Fig. 1 mit den angrenzenden Theilen.

a Pseudokapsel.

$b$ Feinfaseriges Gewebe im Inneren des Kapselsackes.

$c$ Linsenreste (man erkennt in Einzelnen derselben eine periphere fast homogene und eine mehr centrale Parthie mit unregelmässiger Gerinnung). 
这

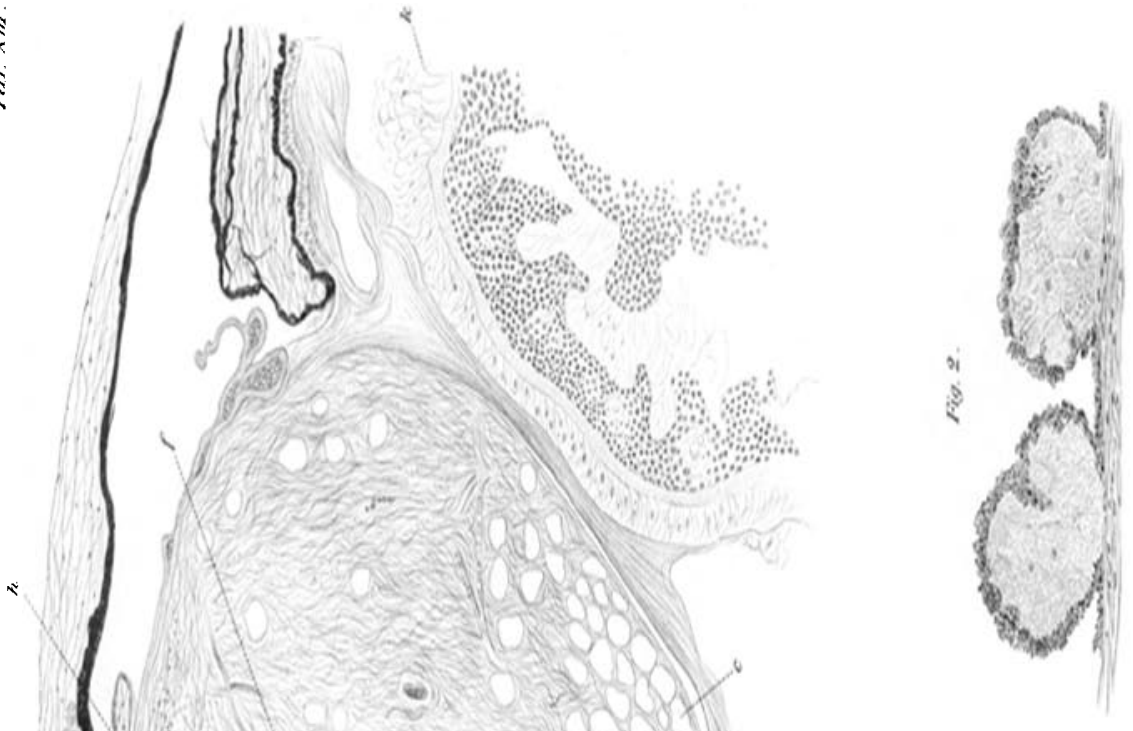

3

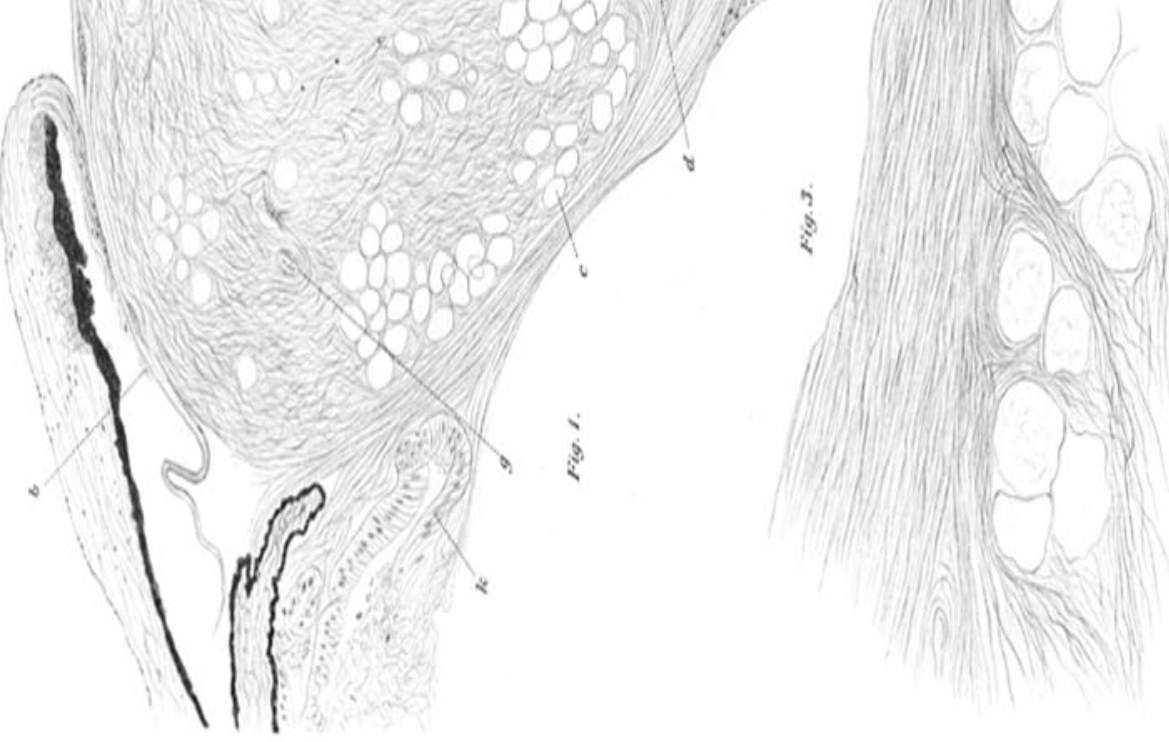

